\title{
STUDY OF THE OPTIMAL HARVESTING CONTROL AND THE OPTIMALITY SYSTEM FOR AN ELLIPTIC PROBLEM
}

\author{
M. DELGADO*, J. A. MONTERO ${ }^{\dagger}$, AND A. SUÁREZ
}

\begin{abstract}
An optimal harvesting problem with concave non-quadratic cost functional and a diffusive degenerate elliptic logistic state equation type is investigated. Under certain assumptions, we prove the existence and uniqueness of an optimal control. A characterization of the optimal control via the optimality system is also derived, which leads to approximate the optimal control.
\end{abstract}

Key words. degenerate logistic equation, singular eigenvalue problems, optimal control, optimality system

AMS subject classifications. 49J20, 49K20, 35J65, 92D25

1. Introduction. In this work we consider the optimal harvesting control of a species whose state is governed by the degenerate elliptic logistic equation, i.e.,

$$
\begin{cases}-\Delta u=(a-f) u^{\alpha}-b u^{\beta} & \text { in } \Omega, \\ u=0 & \text { on } \partial \Omega,\end{cases}
$$

where $\Omega$ is a bounded and regular domain of $\mathbb{R}^{N}, N \geq 1$. Here $a, f$ and $b$ are bounded functions. In particular, $a$ is strictly positive, $b$ is nonnegative and nontrivial, $a-f$ can change sign and $\alpha$ and $\beta$ satisfy

$$
0<\alpha<1, \quad \alpha<\beta .
$$

The solutions of (1.1) can be regarded as the steady states solutions of the corresponding time dependent model. In such case, $u(x)$ stands for the population density and $\Omega$ for the inhabiting area. Since the population is subject to homogeneous Dirichlet boundary conditions, we are assuming that the environment surrounding $\Omega$ is lethal. In such model, the positive function $b(x)$ describes the intro-specific pressure of the species and $a(x)$ represents the growth rate of the species. The function $f(x)$ will be considered non-negative and denotes the distribution of control harvesting of the species by reducing the growth rate. Equation (1.1), under the change of variables $w^{m}=u$, is a particular case of

$$
\begin{cases}-\Delta w^{m}=(a-f) w-b w^{2} & \text { in } \Omega \\ w=0 & \text { on } \partial \Omega\end{cases}
$$

This model was introduced in populations dynamics by Gurtin and MacCamy in [11] for describing the dynamics of biological populations whose mobility depends upon their density. In this context, $m>1$ (nonlinear slow diffusion) means that the diffusion is slower than in the linear case $m=1$, giving rise to more realistic biological results, see [11].

One of the main differences between the degenerate case $(m>1)$ and the nondegenerate one $(m=1)$ is that in the first case the strong maximum principle does not

\footnotetext{
*Dpto. Ecuaciones Diferenciales y Análisis Numérico, Fac. Matemáticas, C/ Tarfia s/n C. P. 41012, Univ. Sevilla, Spain (delgado@numer.us.es),

${ }^{\dagger}$ Dpto. Análisis Matemático, C. P. 18071, Univ. Granada, Spain (jmontero@goliat.ugr.es)

${ }^{\ddagger}$ Dpto. Ecuaciones Diferenciales y Análisis Numérico, Fac. Matemáticas, C/ Tarfia s/n C. P. 41012, Univ. Sevilla, Spain (suarez@numer.us.es)
} 
hold in general. So, unlike the non-degenerate case, three kinds of solutions appear: the trivial solution, the strictly positive solutions (the species can survive in the whole domain) and the nonnegative and nontrivial solutions, which are zero in a region of $\Omega$. This region is called in the literature dead core.

Equation (1.1) has been studied previously for $b=0$ in [1] and [2] and for $b$ strictly positive in [8] and [19] and references therein. However, very little is known in the case that $b$ can vanish in some region. In our knowledge, this problem has been only analyzed in [9] in the particular case $a-f$ equals to a constant. We generalize these results and prove that there exists a maximal nonnegative solution of (1.1), which will be denoted by $u_{f}$. Moreover, when $f$ is such that the function $a-f$ is positive, we show that (1.1) possesses a unique positive solution which is linearly asymptotically stable.

After studying in detail the state equation, our main goal is to analyze the optimal control criteria, that is, maximize the payoff functional

$$
J(f):=\int_{\Omega}\left(\lambda u_{f} h(f)-k(f)\right),
$$

where $h$ and $k$ are regular functions, and $\lambda>0$ will be considered as a parameter. Here, $J$ represents the difference between economic revenue measured by $\int_{\Omega} \lambda u_{f} h(f)$ and the control cost measured by $\int_{\Omega} k(f)$. The parameter $\lambda$ describes the quotient between the price of the species and the cost of the control. This functional includes the special case (quadratic functional)

$$
h(t)=t \quad \text { and } \quad k(t)=t^{2},
$$

which seems to have been introduced in population dynamics in [17] (see also [6], [15] and references therein).

We say that $f \in L_{+}^{\infty}(\Omega)$ is an optimal control if

$$
J(f)=\sup _{g \in L_{+}^{\infty}(\Omega)} J(g) .
$$

This control problem is a generalization of the one studied in detail in [6], [17] and [18], where $\alpha=1, \beta=2, h(t)=t$ and $k(t)=t^{2}$.

In [7], the authors analyzed the case $0<\alpha<1 \leq \beta, b$ strictly positive and the cost functional (1.4) under more restrictive monotony assumptions on functions $h, k$. There, the controls are restricted to the set

$$
\mathcal{D}:=\left\{f \in L_{+}^{\infty}(\Omega): f \leq a \quad \text { a.e. in } \Omega\right\} .
$$

If $f \in \mathcal{D}$, then the maximal solution of (1.1) is strictly positive. In such case, It is proved the existence and uniqueness of optimal control in $\mathcal{D}$ for $\lambda$ sufficiently small.

In this work, we only assume (1.2), $b$ nonnegative and nontrivial and our control space is $L_{+}^{\infty}(\Omega)$. So, $u_{f}$ can have dead cores depending on the control $f \in L_{+}^{\infty}(\Omega)$ chosen. In this framework, we show that there exists an optimal control in $L_{+}^{\infty}(\Omega)$ for any $\lambda>0$. When $\lambda$ is smaller than a determined bound, we can express the optimal control in terms of $u_{f}$ and, if $\lambda$ is small enough then the optimal control is unique. In such case, our assumptions imply that if $f$ is an optimal control, then the dead core for $u_{f}$ is empty. See [20], where a related problem is studied and where the dead core is allowed to exist. 
In order to obtain the uniqueness result, we will use two different ways. Firstly, we follow an argument described in [6] proving that the map $f \mapsto J(f)$ is Fréchet differentiable and strictly concave. The Fréchet derivability of the map $f \mapsto J(f)$ is rather more difficult than in the case $m=1$, because it involves both linear elliptic and eigenvalue problems with potentials which blow-up in a neighborhood of $\partial \Omega$. These difficulties have been solved by using results of singular eigenvalue problems from [4], see also [12]. Secondly, we express the unique optimal control in terms of the solution of the optimality system, and we give an alternative proof of the uniqueness for the optimal control via the optimality system. This is an interesting point in the optimal control problems, because it let us approximate the optimal control by a constructive scheme which provides us a sequence of functions converging to some special solutions of the optimality system. The uniqueness of solution of the optimality system was not considered in [17], but it was studied in [6] in the particular case $m=1$ and the quadratic functional. Here, we present an alternative and shorter proof of the uniqueness, which can be applied to the case studied in [6]. Again, the second alternative presents another technical difficulty that must be overcome: the optimality system is a reaction-diffusion system with a singular reaction term. We present the sub-supersolution method for this kind of systems which provides us an iterative method to approach the solution of the nonlinear system; see [5], [12] for the case of one equation.

An outline of this work is as follows: in Section 2 we introduce some notations and we collect some results concerning the existence and uniqueness of the principal eigenvalue and the corresponding solution for linear elliptic problems with unbounded potentials. In Section 3 we study the equation (1.1). We show the existence of a maximal non-negative solution, and under stronger restrictions on the coefficients, the existence and uniqueness of a positive solution of (1.1). In Section 4, we prove the existence of optimal control for functional $J$ and we show that for $\lambda$ sufficiently small the functional $J$ is Fréchet differentiable and strictly concave. Then, we deduce easily the uniqueness of optimal control. In the last Section we characterize the optimal control. This characterization provides us the optimality system. Finally, we prove the uniqueness of positive solution of the optimality system and an iterative scheme based on alternating monotone sequences, to approach its solution. As it is remarked in recent related works ([16], [17, Remark 4.1]), it is interesting to give conditions to guarantee the convergence of the method to the solution of the optimal control problem.

2. Preliminaries and notations. Let $\Omega$ be a bounded domain in $\mathbb{R}^{N}$ with a smooth boundary $\partial \Omega$. For any $f \in L^{\infty}(\Omega)$ we denote

$$
f_{M}:=\operatorname{ess} \sup f \quad f_{L}:=\operatorname{essinf} f
$$

and define the sets

$$
L_{+}^{\infty}(\Omega):=\left\{f \in L^{\infty}(\Omega): f_{L} \geq 0\right\} \quad L_{-}^{\infty}(\Omega):=\left\{f \in L^{\infty}(\Omega): f_{M} \leq 0\right\} .
$$

Moreover, we denote $C_{0}^{1}(\bar{\Omega})=\left\{u \in C^{1}(\bar{\Omega}): u=0 \quad\right.$ on $\left.\partial \Omega\right\}$ and by $P_{+}$its nonnegative cone, whose interior is

$$
\operatorname{int}\left(P_{+}\right):=\left\{u \in C_{0}^{1}(\bar{\Omega}): u>0 \quad \text { in } \Omega, \partial u / \partial n<0 \quad \text { on } \partial \Omega\right\}
$$


where $n$ is the outward unit normal at $\partial \Omega$.

In this section we primarily consider the singular eigenvalue problem

$$
\begin{cases}-\Delta u+M(x) u=\sigma u & \text { in } \Omega \\ u=0 & \text { on } \partial \Omega\end{cases}
$$

where

$(H M)$

$$
M \in L_{l o c}^{\infty}(\Omega) \quad \text { verifying } \quad M(x) d_{\Omega}(x) \in L^{\infty}(\Omega),
$$

and $d_{\Omega}(x):=\operatorname{dist}(x, \partial \Omega)$.

The following result, whose proof can be found in [13], shows that (2.1) is well defined in $H_{0}^{1}(\Omega)$.

LEMmA 2.1. Let $\varphi \in W_{0}^{1, q}(\Omega)$ for some $1<q<\infty$. Then there exists a constant $C>0$ such that

$$
\left\|\frac{\varphi}{d_{\Omega}}\right\|_{q} \leq C\|\varphi\|_{W^{1, q}(\Omega)}
$$

Although (2.1) is not included in the singular eigenvalue problem studied in [4], we can do some minor changes to the proofs of Theorem 3.4 and Lemma 3.5 in [4] to conclude the existence and uniqueness of the principal eigenvalue of (2.1) and its associated eigenfunction. In the following result, we collect these results and some properties of the principal eigenvalue, see [7].

TheOREM 2.2. Assume that $M$ satisfies $(H M)$. Then there exists a unique principal eigenvalue (i.e., a real eigenvalue with an associated positive eigenfunction $\left.\varphi_{1}(-\Delta+M)\right)$. We denote it by $\sigma_{1}(-\Delta+M)$. Moreover, $\varphi_{1}(-\Delta+M) \in W^{2, p}(\Omega)$ for all $p>1$, and so $\varphi_{1}(-\Delta+M) \in \operatorname{int}\left(P_{+}\right)$. Furthermore,

1. Assume that $M_{i}, i=1,2$ satisfy $(H M)$ and $M_{1} \leq M_{2}$. Then

$$
\sigma_{1}\left(-\Delta+M_{1}\right) \leq \sigma_{1}\left(-\Delta+M_{2}\right) .
$$

2. Assume that $M_{n}, M, n \in \mathbb{N}$ satisfy $(H M)$ with

$$
\int_{\Omega} M_{n} \varphi^{2} \rightarrow \int_{\Omega} M \varphi^{2}, \quad \text { as } n \rightarrow \infty \text { and for all } \varphi \in H_{0}^{1}(\Omega) .
$$

Then,

$$
\sigma_{1}\left(-\Delta+M_{n}\right) \rightarrow \sigma_{1}(-\Delta+M) \quad \text { as } n \rightarrow \infty .
$$

In the particular case $M \equiv 0$, we denote $\sigma_{1}:=\sigma_{1}(-\Delta)$ and $\varphi_{1}=\varphi_{1}(-\Delta)$ normalized such that $\left\|\varphi_{1}\right\|_{\infty}=1$.

When $M$ verifies $(H M)$, the following strong maximum principle is satisfied.

Lemma 2.3. Let $u \in W^{2, p}(\Omega) \cap C^{1}(\bar{\Omega}), p>1$, be such that $u \geq 0$ in $\Omega, u \neq 0$ and

$$
(-\Delta+M) u \geq 0 \quad \text { a.e. in } \Omega, \quad u \geq 0 \quad \text { on } \partial \Omega .
$$

Then $u(x)>0$ for all $x \in \Omega$ and $(\partial u / \partial n)\left(x_{0}\right)<0$ for all $x_{0} \in \partial \Omega$ where $u\left(x_{0}\right)=0$.

Proof. Assume there exists $x_{0} \in \Omega$ such that $u\left(x_{0}\right)=0$. By hypothesis, we can take $x_{1} \in \Omega$ where $u\left(x_{1}\right)>0$ and a subdomain regular $\Omega_{1} \subset \Omega$ such that $x_{0}, x_{1} \in \Omega_{1}$. But $M \in L^{\infty}\left(\Omega_{1}\right)$, and so the strong maximum principle leads us to a contradiction. 
On the other hand, applying the Lemma 3.6 in [4] with $\rho(s)=s^{-1}$, we get that $(\partial u / \partial n)\left(x_{0}\right)<0$ for all $x_{0} \in \partial \Omega$ such that $u\left(x_{0}\right)=0$.

The following technical result will help us to prove the positivity of the some principal eigenvalue.

Proposition 2.4. Assume that $M$ satisfies $(H M)$ and that there exists $\varphi \in$ $W_{\text {loc }}^{2, p}(\Omega) \cap C_{0}^{0}(\bar{\Omega}), p>N$, such that $\varphi>0$ in $\Omega$ and for all subdomain $\Omega^{\prime} \subset \bar{\Omega}^{\prime} \subset \Omega$ it holds $(-\Delta+M) \varphi:=F$ with $F_{L}>0$ in $\Omega^{\prime}$. Then, $\sigma_{1}(-\Delta+M)>0$.

Proof. From Krein-Rutman theorem, it is well-known that if $-\Delta+M$ satisfies the strong maximum principle in $\Omega$, then $\sigma_{1}(-\Delta+M)>0$. Let $v \in W^{2, p}(\Omega) \cap C^{1}(\bar{\Omega})$ be such that $v \neq 0$, and

$$
(-\Delta+M) v \geq 0 \quad \text { in a.e. } \Omega, \quad v \geq 0 \quad \text { on } \partial \Omega .
$$

We have to prove that $v>0$ in $\Omega$ and $\partial v / \partial n(x)<0$ for all $x \in \partial \Omega$ such that $v(x)=0$. For each $\epsilon>0$ and $K>0$, we define

$$
w:=v+\epsilon+\epsilon K \varphi \in C^{0}(\bar{\Omega}) .
$$

and so, for any $\epsilon>0$, there exists $\gamma(\epsilon)>0$ such that $w>0$ in $\Omega_{\epsilon}:=\left\{x \in \Omega: d_{\Omega}(x)<\right.$ $\gamma(\epsilon)\}$. Moreover

$$
(-\Delta+M) w \geq \epsilon(M+K F)>0 \quad \text { a.e. in } \Omega \backslash \bar{\Omega}_{\epsilon},
$$

for $K$ sufficiently large. Moreover, since $\varphi$ is a strict supersolution in $\Omega \backslash \bar{\Omega}_{\epsilon}$, we can apply the Corollary 2.4 in [3] and we obtain that $w>0$ in $\Omega \backslash \bar{\Omega}_{\epsilon}$. Thus, we get that $w>0$ in $\Omega \backslash \bar{\Omega}_{\epsilon}$. Hence, $w>0$ in $\Omega$ for all $\epsilon>0$, and we obtain that $v \geq 0$ in $\Omega$. Now, it suffices to apply Lemma 2.3 .

Given $M$ verifying $(H M)$ and $f \in L^{\infty}(\Omega)$ we consider the problem

$$
\begin{cases}-\Delta u+M(x) u=f & \text { in } \Omega, \\ u=0 & \text { on } \partial \Omega .\end{cases}
$$

Observe that by Lemma 2.1, (2.4) is well defined in $H_{0}^{1}(\Omega)$. The following result (whose proof can be found in [7]) shows that (2.4) possesses a unique solution in $C_{0}^{1}(\bar{\Omega})$, it provides us of an useful estimate and properties of the solution.

Theorem 2.5. Assume that $M$ satisfies $(H M)$ and $\sigma_{1}(-\Delta+M)>0$. Then, there exists a unique solution $u \in C^{1, \kappa}(\bar{\Omega})$, for some $\kappa \in(0,1)$ of $(2.4)$. Moreover, there exists a constant $K>0$ (independent of $f$ ) such that

$$
\|u\|_{C^{1, \kappa}(\bar{\Omega})} \leq K\|f\|_{\infty}
$$

Furthermore, the following properties hold:

1. Consider $f_{i} \in L^{\infty}(\Omega), i=1,2$ with $f_{1} \leq f_{2}$ and let $u_{i}, i=1,2$ be the respective solutions of (2.4). Then, $u_{1} \leq u_{2}$.

2. Assume that $M_{i}, i=1,2$ satisfy $(H M), \sigma_{1}\left(-\Delta+M_{1}\right)>0$ and $M_{1} \leq M_{2}$. Let $u_{i}, i=1,2$ be the respective solutions of (2.4) with $f \in L_{+}^{\infty}(\Omega)$. Then, $u_{2} \leq u_{1}$.

Note: Similar results to the previous ones have been obtained in [12] when $M \in$ $C^{1}(\Omega), M d_{\Omega}^{\gamma} \in L^{\infty}(\Omega)$ for $\gamma \in(0,2)$ and the operator is not necessarily selfadjoint. 
3. The state equation. Consider the equation

$$
\begin{cases}-\Delta u=(a-f) u^{\alpha}-b u^{\beta} & \text { in } \Omega, \\ u=0 & \text { on } \partial \Omega,\end{cases}
$$

and assume that

$$
\begin{gathered}
0<\alpha<1, \quad \alpha<\beta, \quad a, b \in L_{+}^{\infty}(\Omega) \backslash\{0\}, \quad f \in L^{\infty}(\Omega), \\
a_{L}>0,(a-f)_{M}>0 .
\end{gathered}
$$

Observe that if $(a-f)_{M} \leq 0$ then, by the maximum principle, (3.1) does not possess nonnegative and nontrivial solution. This justifies the hypothesis $(a-f)_{M}>0$. In order to study (3.1), we consider the porous medium equation

$$
\begin{cases}-\Delta w=\mu w^{\alpha} & \text { in } \Omega, \\ w=0 & \text { on } \partial \Omega,\end{cases}
$$

where $\mu \in \mathbb{R}$. It holds:

Lemma 3.1. Assume $0<\alpha<1$. The porous medium equation (3.2) has a nontrivial and nonnegative solution if, and only if, $\mu>0$. If $\mu>0$, there exists a unique solution, denoted $w_{\mu}$, which is strictly positive and $w_{\mu} \in C^{2, \alpha}(\bar{\Omega})$. Moreover, it verifies

$$
\epsilon_{0} \varphi_{1} \leq w_{\mu} \leq K_{0} e, \quad \text { in } \Omega,
$$

where $e$ is the unique positive solution of

$$
-\Delta e=1 \quad \text { in } \Omega, \quad e=0 \quad \text { on } \partial \Omega,
$$

and $\epsilon_{0}^{1-\alpha}=\mu / \sigma_{1}, K_{0}^{1-\alpha}=\mu\|e\|_{\infty}^{\alpha}$.

The results of existence and uniqueness of positive solution of (3.2) are wellknown, see [1] for instance. The estimate (3.3) can be obtained easily by the subsupersolution method.

The following result shows that (3.1) has a maximal nonnegative solution.

TheOREM 3.2. Assume (H1). There exists a unique maximal nonnegative solution $u_{f}$ of (3.1). Moreover, by elliptic regularity $u_{f} \in W^{2, p}(\Omega)$, for all $p>1$, and so $u_{f} \in C^{1, \kappa}(\bar{\Omega})$, with $0<\kappa \leq 1-N / p$. Furthermore, we have the following a priori bound,

$$
\left\|u_{f}\right\|_{\infty} \leq\left((a-f)_{M}\|e\|_{\infty}\right)^{1 /(1-\alpha)} .
$$

Finally, the map $f \mapsto u_{f}$ is non-increasing.

Proof. Let $u$ be a weak solution of (3.1), then by (H1) and elliptic regularity it follows that $u \in C_{0}^{1}(\bar{\Omega})$. So, there exists $K>0$ sufficiently large such that

$$
u \leq K e \quad \text { in } \Omega,
$$

and the pair $(u, K e)$ is a sub-supersolution of $(3.2)$ with $\mu=(a-f)_{M}$. By the uniqueness of positive solution of (3.2) it follows that

$$
u \leq w_{(a-f)_{M}} .
$$

The existence of positive a priori bounds and that $u \equiv 0$ is a solution of equation (3.1) imply the existence of a nonnegative maximal solution of (3.1). By (3.3) we get the bound (3.4). 
Let $f_{1}, f_{2} \in L^{\infty}(\Omega)$ be such that $f_{1} \leq f_{2}$. It is clear that the pair $\left(u_{f_{2}}, K e\right)$ is a sub-supersolution of (3.1) for $f=f_{1}$ for $K>0$ sufficiently large. So, there exists a solution $u$ such that $u_{f_{2}} \leq u \leq K e$. The maximality of $u_{f_{1}}$ completes the proof. 口

Note: From (3.4) we get, for each maximal nonnegative solution of (3.1), a uniform upper bound, i.e.,

$$
u_{f} \leq\left((a-f)_{M}\|e\|_{\infty}\right)^{1 /(1-\alpha)} \leq\left(a_{M}\|e\|_{\infty}\right)^{1 /(1-\alpha)}:=\mathcal{K},
$$

for any $f \in L_{+}^{\infty}(\Omega)$.

Observe that $u_{f}$ would be eventually the trivial solution. The following result shows that this can not occur in a subset of $L^{\infty}(\Omega)$. We define

$$
\mathcal{C}:=\left\{f \in L^{\infty}(\Omega):(a-f)_{L}>0\right\} .
$$

In the following result we prove the existence and uniqueness of positive solution of (3.1) when $f \in \mathcal{C}$.

Proposition 3.3. Assume $(H 1)$ and let $f \in \mathcal{C}$. Then, there exists a unique nontrivial and nonnegative solution, $u_{f}$, of (3.1). Moreover, $u_{f}$ is strictly positive, in fact,

$$
\epsilon_{f} \varphi_{1} \leq u_{f}, \quad \text { in } \Omega
$$

where $\epsilon_{f}$ satisfies

$$
\epsilon_{f}^{1-\alpha} \sigma_{1}+\epsilon_{f}^{\beta-\alpha} b_{M}=(a-f)_{L} .
$$

Moreover, $u_{f}$ is linearly asymptotically stable, i.e.,

$$
\sigma_{1}\left(-\Delta+M_{f}\right)>0
$$

where

$$
M_{f}:=-\alpha(a-f) u_{f}^{\alpha-1}+\beta b u_{f}^{\beta-1} .
$$

Furthermore, the map $f \in \mathcal{C} \mapsto u_{f}$ is continuous.

Note: Observe that by $(H 1),(3.7)$ possesses a unique positive solution.

Proof. For the existence of solution, it is not hard to show that $\left(\epsilon_{f} \varphi_{1}, w_{(a-f)_{M}}\right)$ is a sub-supersolution of (3.1) for $\epsilon_{f}>0$ defined in (3.7).

Observe that by the strong maximum principle for $f \in \mathcal{C}$, any nontrivial and nonnegative solution $u$ of (3.1) is strictly positive, this means that $u \in \operatorname{int}\left(P_{+}\right)$.

The uniqueness of positive solution follows as in Theorem 1 of [9] and the continuity of the map $f \mapsto u_{f}$ as in Theorem 3.3 of [7].

It remains to prove (3.8). Firstly observe that $M_{f}$ satisfies $(H M)$. Indeed, by (3.6), there exists a positive constant $C$ (independent of $f$ ) such that

$$
C \epsilon_{f} d_{\Omega} \leq u_{f} \quad \text { in } \Omega .
$$

Thus, since $\alpha<1$, we have that

$$
\begin{aligned}
\left|M_{f}\right| d_{\Omega} & =u_{f}^{\alpha-1} d_{\Omega}\left|-\alpha(a-f)+\beta b u_{f}^{\beta-\alpha}\right| \\
& \leq C^{\alpha-1} \epsilon_{f}^{\alpha-1} d_{\Omega}^{\alpha-1} d_{\Omega}\left|-\alpha(a-f)+\beta b u_{f}^{\beta-\alpha}\right| \leq K,
\end{aligned}
$$

for some $K>0$. Therefore, $M_{f}$ satisfies $(H M)$ and $\sigma_{1}\left(-\Delta+M_{f}\right)$ is well defined. Observe that $u_{f}^{\alpha} \in W_{l o c}^{2, p}(\Omega) \cap C_{0}^{0}(\bar{\Omega})$ for all $p>1$ and it satisfies

$$
\left(-\Delta+M_{f}\right)\left(u_{f}^{\alpha}\right)=\alpha(1-\alpha) u_{f}^{\alpha-2}\left|\nabla u_{f}\right|^{2}+(\beta-\alpha) b u_{f}^{\alpha+\beta-1}>0, \quad \text { in } \Omega,
$$

and thus, we can apply Proposition 2.4 and conclude that $\sigma_{1}\left(-\Delta+M_{f}\right)>0$. 
4. Existence and uniqueness of optimal control. For $\lambda>0$ we consider the functional $J: L_{+}^{\infty}(\Omega) \mapsto \mathbb{R}$,

$$
J(g):=\int_{\Omega}\left(\lambda h(g) u_{g}-k(g)\right),
$$

where $h \in C^{1}\left(\mathbb{R}^{+} ; \mathbb{R}^{+}\right), k \in C^{2}\left(\mathbb{R}^{+} ; \mathbb{R}^{+}\right) ; h(s)=0$ if and only if $s=0$, and $k(s)=0$ if and only if $s=0$. Function $h$ is concave and $k$ is a strictly convex function satisfying $k^{\prime \prime}(s) \geq k_{0}>0$ for some $k_{0}$. Note that $h^{\prime}, k^{\prime}$ are Lipschitz continuous functions on bounded set. We assume:

$$
\lim _{t \rightarrow 0} \frac{k(t)}{h(t)}=0, \quad \quad \lim _{t \rightarrow+\infty} \frac{k(t)}{h(t)}=+\infty .
$$

Observe that the particular case $h(t)=t$ and $k(t)=t^{2}$, studied in [6], [15], [17] and [18], is in the setting of our functional. Also, we remove some hypotheses of monotonous type involving functions $k$ and $h$ considered in [7]. The idea will be to show that the integrand of functional $J(f)$ must be positive if $f$ is an optimal control.

In the first part of this Section we want to prove the existence of the optimal control under hypothesis $(H 2)$. Firstly, we prove that the optimal controls are bounded.

Lemma 4.1. Assume (H2). If $f \in L_{+}^{\infty}(\Omega)$ is an optimal control, then

$$
\lambda u_{f}(x) h(f(x)) \geq k(f(x)), \text { a.e. in } \Omega .
$$

Moreover, if $f \in L_{+}^{\infty}(\Omega)$ is an optimal control, then

$$
0 \leq f \leq T_{\lambda}
$$

where

$$
T_{\lambda}:=\sup \left\{t \in \mathbb{R}^{+}: \frac{k(t)}{h(t)}=\lambda \mathcal{K}\right\},
$$

and $\mathcal{K}$ is the uniform bound defined in (3.5).

Note: By the hypotheses imposed to $h$ and $k$ and $(H 2)$, it follows that $T_{\lambda}>0$ and that $T_{\lambda} \rightarrow 0$ as $\lambda \downarrow 0$.

Proof. Suppose that $f \in L_{+}^{\infty}(\Omega)$ be an optimal control and (4.1) is not true. Then, $\exists \Omega_{1} \subset \Omega$ with $\left|\Omega_{1}\right|>0$ (positive measure) such that

$$
\lambda u_{f}(x) h(f(x))<k(f(x)), \quad \forall x \in \Omega_{1}
$$

Now, by defining a new control $\bar{f}$ as

$$
\bar{f}(x)= \begin{cases}f(x) & \text { if } x \in \Omega \backslash \Omega_{1}, \\ 0 & \text { if } x \in \Omega_{1},\end{cases}
$$

and taking into account that $u_{\bar{f}} \geq u_{f}$ in $\Omega$, we obtain

$$
\begin{aligned}
& J(f)=\int_{\Omega_{1}} \lambda u_{f}(x) h(f(x))-k(f(x))+\int_{\Omega \backslash \Omega_{1}} \lambda u_{f}(x) h(f(x))-k(f(x)) \\
& <\int_{\Omega \backslash \Omega_{1}} \lambda u_{f}(x) h(f(x))-k(f(x)) \leq \int_{\Omega \backslash \Omega_{1}} \lambda u_{\bar{f}}(x) h(f(x))-k(f(x)) \\
& =\int_{\Omega \backslash \Omega_{1}} \lambda u_{\bar{f}}(x) h(\bar{f}(x))-k(\bar{f}(x))=J(\bar{f}) .
\end{aligned}
$$


But $f$ is an optimal control. So, previous inequality shows that (4.2) is absurd. Also, that $f \leq T_{\lambda}$ follows from the definition of $T_{\lambda}$, Theorem 3.2 and (4.1).

ThEOREM 4.2. Assume (H2). There exists an optimal control, i.e., $f \in L_{+}^{\infty}(\Omega)$ such that

$$
J(f)=\sup _{g \in L_{+}^{\infty}(\Omega)} J(g)
$$

Moreover, the benefit is positive, i.e., $\sup _{g \in L_{+}^{\infty}(\Omega)} J(g)>0$.

Proof. By (3.5) and Lemma 4.1, it follows that

$$
s:=\sup _{g \in L_{+}^{\infty}(\Omega)} J(g)<+\infty,
$$

and so, there exists a maximizing sequence $f_{n} \in L_{+}^{\infty}(\Omega)$. By a similar reasoning to the used in the previous Lemma, we can suppose that $0 \leq f_{n} \leq T_{\lambda}$. Then, there exists a subsequence, relabelled by $f_{n}$, such that

$$
f_{n} \rightarrow f \in\left[0, T_{\lambda}\right] \quad \text { in } L^{2}(\Omega) .
$$

By (3.5), we can prove that

$$
u_{f_{n}} \rightarrow u_{*} \quad \text { in } H_{0}^{1}(\Omega),
$$

where $u_{*}$ is a positive solution of (3.1) (possibly no the maximal positive solution). In any case, we have $u_{f} \geq u_{*}$.

Now, taking into account the concavity of the functions $h$ and $-k$, it follows that

$$
J(f) \geq \limsup \int_{\Omega} \lambda h\left(f_{n}\right) u_{f_{n}}-k\left(f_{n}\right)=s,
$$

and so the existence of an optimal control.

The optimal benefit is positive by following an argument like the used in [7]. In fact, it is clear, from the asymptotic properties of the functions $h$ and $k$, that $J(\epsilon)>0$ by taking $\epsilon \in \mathbb{R}^{+}$small enough.

Now, we are going to prove that, for $\lambda$ sufficiently small, there exists a unique optimal control. For that we will use the argument described in Section 6 in [6]. In summary, by Lemma 4.1 we know that the optimal controls belong to a convex, $\left[0, T_{\lambda}\right]$. Moreover, we will show that $J$ is Fréchet continuously differentiable and strictly concave in $\left[0, T_{\lambda}\right]$. Hence, the uniqueness of optimal control is a direct consequence. The first step is the following result which provides us the Gâteaux derivative of the map $f \in \mathcal{C} \mapsto u_{f} \in \operatorname{int}\left(P_{+}\right)$. Its proof is similar to Lemma 3.5 in [7], and so we omit it.

Lemma 4.3. Let $f \in \mathcal{C}, g \in L^{\infty}(\Omega)$, and $\epsilon \simeq 0$ be such that $f+\epsilon g \in \mathcal{C}$. Then,

$$
\frac{u_{f+\epsilon g}-u_{f}}{\epsilon} \rightarrow \xi_{f, g} \quad \text { in } H_{0}^{1}(\Omega) \text { as } \epsilon \rightarrow 0,
$$

where $\xi_{f, g}$ is the unique solution of

$$
\begin{cases}-\Delta \xi+M_{f}(x) \xi=-g u_{f}^{\alpha} & \text { in } \Omega, \\ \xi=0 & \text { on } \partial \Omega .\end{cases}
$$


Observe that (4.4) has a unique solution because $\sigma_{1}\left(-\Delta+M_{f}\right)>0$ (see (3.8)) and Theorem 2.5 .

Now, we can prove (see Proposition 4.4 in [7])

Proposition 4.4. Let $J: \mathcal{C} \subset L^{\infty}(\Omega) \mapsto \mathbb{R}$ be. Then $J$ is Fréchet continuously differentiable and

$$
J^{\prime}(f)(g)=\int_{\Omega}\left(\lambda h^{\prime}(f) u_{f}-\lambda u_{f}^{\alpha} P_{f}-k^{\prime}(f)\right) g, \quad \forall f \in \mathcal{C}, \forall g \in L^{\infty}(\Omega),
$$

where for any $f \in \mathcal{C}, P_{f} \in C_{0}^{1}(\bar{\Omega})$ is the unique solution of

$$
\begin{cases}-\Delta P_{f}+M_{f}(x) P_{f}=h(f) & \text { in } \Omega, \\ P_{f}=0 & \text { on } \partial \Omega,\end{cases}
$$

and $M_{f}$ is defined in (3.9).

Note: Since $M_{f}$ satisfies $(H M)$ and by (3.8), it follows from Theorem 2.5 the existence and uniqueness of $P_{f} \in C_{0}^{1}(\bar{\Omega})$.

Observe that by Note following Lemma 4.1, there exists $\lambda_{0}>0$ such that

$$
a_{L}>T_{\lambda} \text { for } \lambda<\lambda_{0} .
$$

Following the argument of Theorem 3.1 in [17] (using now (4.7) and Proposition 4.4) we obtain.

Corollary 4.5. Assume (H2). Let $f \in L_{+}^{\infty}(\Omega)$ be a optimal control. Then for $\lambda<\lambda_{0}$,

$$
k^{\prime}(f)=\lambda\left(h^{\prime}(f) u_{f}-u_{f}^{\alpha} P_{f}\right)^{+} .
$$

In order to prove that $J$ is strictly concave in $\left[0, T_{\lambda}\right]$, we will show that maps involved in $J^{\prime}$ are Lipschitz continuous. This result was proven in [7] when $\beta \geq 1$. Since the Lipschitz character of the maps involved is crucial in this work (see for example the proof of Lemma 5.4), we present a complete proof of this result for the reader's convenience.

TheOREM 4.6. Assume (H2). There exists $\Lambda>0$ such that for $0<\lambda<\Lambda$ the maps

$$
f \in\left[0, T_{\lambda}\right] \mapsto u_{f}, P_{f}, u_{f}^{\alpha} P_{f} \in L^{\infty}(\Omega)
$$

are Lipschitz continuous, with the Lipschitz constants independent of $\lambda$.

Proof. Let $f, g \in\left[0, T_{\lambda}\right]$ be, by the monotony of the map $f \mapsto u_{f}$, it follows that $u_{T_{\lambda}} \leq u_{f}, u_{g} \leq u_{0}$. Moreover, for $\lambda<\lambda_{0}$ (defined in (4.7)), $u_{T_{\lambda}}>0$ and so,

$$
0<u_{T_{\lambda}} \leq u_{f}, u_{g} \leq u_{0}
$$

for $\lambda<\lambda_{0}$. Hereafter, we take $\lambda<\lambda_{0}$. By the Mean Value Theorem,

$$
\begin{gathered}
u_{f}^{\alpha}-u_{g}^{\alpha}=\alpha \theta^{\alpha-1}(f, g)\left(u_{f}-u_{g}\right), \quad u_{f}^{\beta}-u_{g}^{\beta}=\beta \eta^{\beta-1}(f, g)\left(u_{f}-u_{g}\right) \quad \text { with } \\
0<u_{T_{\lambda}} \leq \min \left\{u_{f}, u_{g}\right\} \leq \theta(f, g), \eta(f, g) \leq \max \left\{u_{f}, u_{g}\right\} \leq u_{0} .
\end{gathered}
$$

Let $w:=u_{f}-u_{g}$ be. Then, $w$ satisfies

$$
\begin{cases}(-\Delta+N(f, g)) w=(g-f) u_{g}^{\alpha} & \text { in } \Omega \\ w=0 & \text { on } \partial \Omega\end{cases}
$$


where

$$
N(f, g):=-\alpha(a-f) \theta^{\alpha-1}(f, g)+\beta b \eta^{\beta-1}(f, g) .
$$

Using $f \geq 0$ and (4.8), it follows that

$$
N(f, g) \geq-\alpha a \theta^{\alpha-1}(f, g)+\beta b \eta^{\beta-1}(f, g) \geq m_{\lambda},
$$

where

$$
m_{\lambda}:= \begin{cases}-\alpha a u_{T_{\lambda}}^{\alpha-1}+b \beta u_{T_{\lambda}}^{\beta-1} & \text { if } \beta \geq 1 \\ -\alpha a u_{T_{\lambda}}^{\alpha-1}+b \beta u_{0}^{\beta-1} & \text { if } \beta<1\end{cases}
$$

It is not hard to show that $m_{\lambda}$ satisfies $(H M)$. Moreover, we claim that as $\lambda \downarrow 0$,

$$
\int_{\Omega} m_{\lambda} \varphi^{2} \rightarrow \int_{\Omega}\left(-\alpha a u_{0}^{\alpha-1}+b \beta u_{0}^{\beta-1}\right) \varphi^{2} \quad \forall \varphi \in H_{0}^{1}(\Omega),
$$

Indeed, for $\varphi \in H_{0}^{1}(\Omega)$ and using (3.10) we have

$$
\int_{\Omega}\left(u_{T_{\lambda}}^{\alpha-1}-u_{0}^{\alpha-1}\right) \varphi^{2}=\int_{\Omega}\left(u_{T_{\lambda}}^{\alpha}-u_{T_{\lambda}} u_{0}^{\alpha-1}\right) \frac{\varphi}{u_{T_{\lambda}}} \varphi \leq C \epsilon_{T_{\lambda}}^{-1}\left\|u_{T_{\lambda}}^{\alpha}-u_{T_{\lambda}} u_{0}^{\alpha-1}\right\|_{\infty} \int_{\Omega} \frac{\varphi}{d_{\Omega}} \varphi,
$$

where $\epsilon_{T_{\lambda}}$ is defined in (3.7). By the continuity of the map $f \mapsto u_{f}$, Lemma 2.1 and the fact that $\epsilon_{T_{\lambda}}$ does not tend to 0 as $\lambda \downarrow 0$, we obtain that

$$
\int_{\Omega}\left(u_{T_{\lambda}}^{\alpha-1}-u_{0}^{\alpha-1}\right) \varphi^{2} \rightarrow 0 \quad \text { as } \lambda \downarrow 0 .
$$

Reasoning similarly with the other terms, (4.10) is proved. So, by Theorem 2.2 we obtain that

$$
\sigma_{1}(-\Delta+N(f, g)) \geq \sigma_{1}\left(-\Delta+m_{\lambda}\right) \rightarrow \sigma_{1}\left(-\Delta-\alpha a u_{0}^{\alpha-1}+b \beta u_{0}^{\beta-1}\right)>0
$$

as $\lambda \downarrow 0$. This last inequality follows by (3.8) because $f \equiv 0 \in \mathcal{C}$. Hence, using the monotony of the map $\lambda \mapsto T_{\lambda}$, there exists $\lambda_{1}>0$ such that

$$
N(f, g) \geq m_{\lambda} \geq m_{\lambda_{1}}
$$

and

$$
\sigma_{1}(-\Delta+N(f, g)) \geq \sigma_{1}\left(-\Delta+m_{\lambda_{1}}>0, \quad \text { for } \lambda<\lambda_{1}\right.
$$

So, by (4.12) we get

$$
\left(-\Delta+m_{\lambda_{1}}\right) w \leq(g-f) u_{g}^{\alpha},
$$

and hence, using (4.13), Theorem 2.5 and (3.5), it follows that

$$
\left\|u_{f}-u_{g}\right\|_{\infty}=\|w\|_{\infty} \leq\|w\|_{C^{1}(\bar{\Omega})} \leq C\|f-g\|_{\infty} .
$$

This shows that the map $f \mapsto u_{f}$ is Lipschitz.

Now, take $f \in\left[0, T_{\lambda}\right]$. Using the monotony of the map $f \mapsto u_{f}$, we have that

$$
M_{f} \geq m_{\lambda_{1}} .
$$


Thus, by Theorem 2.5 we obtain that

$$
P_{f} \leq \mathcal{P} \quad \text { in } \Omega,
$$

where $\mathcal{P} \in C_{0}^{1}(\bar{\Omega})$ is the unique solution of

$$
\left\{\begin{array}{rll}
-\Delta u+m_{\lambda_{1}} u & =T & \text { in } \Omega, \\
u & =0 & \text { on } \partial \Omega,
\end{array}\right.
$$

and $T:=\max _{r \in\left[0, T_{\lambda_{1}}\right]} h(r)$.

We will prove now that the map $f \mapsto P_{f}$ is Lipschitz. Let $f, g \in\left[0, T_{\lambda}\right]$ and $z:=P_{f}-P_{g}$ be. Then $z$ satisfies

$$
-\Delta z+M_{f} z=T(f, g), \quad \text { in } \Omega, \quad z=0 \quad \text { on } \partial \Omega,
$$

where

$T(f, g)=h(f)-h(g)+P_{g}\left[\alpha(a-f)\left(u_{f}^{\alpha-1}-u_{g}^{\alpha-1}\right)-\beta b\left(u_{f}^{\beta-1}-u_{g}^{\beta-1}\right)\right]+\alpha(g-f) P_{g} u_{g}^{\alpha-1}$.

Applying again the Mean Value Theorem, we get

$$
\begin{gathered}
u_{f}^{\alpha-1}-u_{g}^{\alpha-1}=(\alpha-1) \xi^{\alpha-2}(f, g)\left(u_{f}-u_{g}\right), \\
u_{f}^{\beta-1}-u_{g}^{\beta-1}=(\beta-1) \eta^{\beta-2}(f, g)\left(u_{f}-u_{g}\right) \\
0<u_{T_{\lambda}} \leq \min \left\{u_{f}, u_{g}\right\} \leq \xi(f, g), \eta(f, g) \leq \max \left\{u_{f}, u_{g}\right\} \leq u_{0} .
\end{gathered}
$$

Hence,

$T(f, g)=h(f)-h(g)+P_{g}\left[\alpha(\alpha-1)(a-f) \xi^{\alpha-2}-\beta(\beta-1) b \eta^{\beta-2}\right]\left(u_{f}-u_{g}\right)+\alpha(g-f) P_{g} u_{g}^{\alpha-1}$.

By a similar argument to the used in the proof of (4.14), we obtain

$$
\left\|P_{f}-P_{g}\right\|_{\infty}=\|z\|_{\infty} \leq C\|T(f, g)\|_{\infty} .
$$

Since $\mathcal{P} \in C_{0}^{1}(\bar{\Omega})$, it follows that

$$
|\mathcal{P}(x)| \leq d_{\Omega}(x)\|\mathcal{P}\|_{C^{1}(\bar{\Omega})} .
$$

So, using (3.10), (4.16) and (4.19), we obtain

$$
\begin{aligned}
\left\|(f-g) P_{g} u_{g}^{\alpha-1}\right\|_{\infty} & \leq C\|f-g\|_{\infty}\left\|P_{g} u_{T_{\lambda}}^{\alpha-1}\right\|_{\infty} \leq C\|f-g\|_{\infty}\left\|\mathcal{P} d_{\Omega}^{\alpha-1}\right\|_{\infty} \\
& \leq C\|f-g\|_{\infty}\left\|d_{\Omega}^{\alpha}\right\|_{\infty}\|\mathcal{P}\|_{C^{1}(\bar{\Omega})} \leq C\|f-g\|_{\infty},
\end{aligned}
$$

with $C$ independent of $f$ and $g$.

On the other hand, since $u_{f}-u_{g} \in C_{0}^{1}(\bar{\Omega})$ and using (4.14), (4.16), (4.17) and (4.19)

$$
\begin{aligned}
\left\|(a-f) P_{g} \xi^{\alpha-2}\left(u_{f}-u_{g}\right)\right\|_{\infty} & \leq C\left\|\mathcal{P} \xi^{\alpha-2}\left(u_{f}-u_{g}\right)\right\|_{\infty} \\
& \leq C\|\mathcal{P}\|_{C^{1}(\bar{\Omega})}\left\|d_{\Omega}^{\alpha}\right\|_{\infty}\left\|u_{f}-u_{g}\right\|_{C^{1}(\bar{\Omega})} \leq C\|f-g\|_{\infty}
\end{aligned}
$$

with $C$ independent of $f$ and $g$.

Analogously it can be treated the term $P_{g} \eta^{\beta-2}\left(u_{f}-u_{g}\right)$. Then, since $h$ is Lipschitz in $\left[0, T_{\lambda}\right]$ and by (4.18), it follows that the map $f \mapsto P_{f}$ is Lipschitz.

Let $f, g \in\left[0, T_{\lambda}\right]$ be. By (4.8), we have

$$
\left\|\left(u_{f}^{\alpha}-u_{g}^{\alpha}\right) P_{f}\right\|_{\infty}=\left\|\alpha \xi^{\alpha-1} P_{f}\left(u_{f}-u_{g}\right)\right\|_{\infty} \leq C\|\mathcal{P}\|_{C^{1}(\bar{\Omega})}\|f-g\|_{\infty} \leq C\|f-g\|_{\infty},
$$


and so,

$$
\left\|u_{f}^{\alpha} P_{f}-u_{g}^{\alpha} P_{g}\right\|_{\infty} \leq\left\|\left(u_{f}^{\alpha}-u_{g}^{\alpha}\right) P_{f}\right\|_{\infty}+\left\|u_{g}^{\alpha}\left(P_{f}-P_{g}\right)\right\|_{\infty} \leq C\|f-g\|_{\infty} .
$$

This completes the proof.

We can conclude the main result about uniqueness of optimal control of this Section:

Theorem 4.7. Assume (H2). Then, there exists $\Lambda_{0}>0$ such that if $\lambda<\Lambda_{0}$ there exists a unique optimal control.

5. The optimality system and the approximation to the optimal control. In this section, we deduce the optimality system in the special case $h(t)=t$ and $k(t)=t^{2}$, which satisfy clearly $(H 2)$. The optimality system will be used to demonstrate the uniqueness of the optimal control in a different way and provides an iterative method to approach it. In this case, we know that

$$
T_{\lambda}=\lambda \mathcal{K} \quad \text { and } \quad \lambda_{0}=\frac{a_{L}}{\mathcal{K}},
$$

where $\mathcal{K}$ is defined in (3.5). Moreover, by Corollary 4.5, for $\lambda<\lambda_{0}$, if $f$ is an optimal control, then

$$
f=\frac{\lambda}{2} u_{f}\left(1-u_{f}^{\alpha-1} P_{f}\right)^{+} .
$$

Let $\psi$ be the unique positive solution of

$$
\begin{cases}-\Delta \psi+m_{\lambda_{1}} \psi=\mathcal{K} & \text { in } \Omega, \\ \psi=0 & \text { on } \partial \Omega,\end{cases}
$$

where $m_{\lambda_{1}}$ is defined in (4.9) and satisfies (4.12) and (4.13). So, if $f$ is an optimal control it follows by Lemma 4.1 that $f \in[0, \lambda \mathcal{K}]$. On the other hand, by $(4.15)$ and Theorem 2.5, we get that

$$
P_{f} \leq \lambda \psi \quad \text { for } \lambda \leq \lambda_{1} .
$$

As consequence of (5.3) we obtain (see Proposition 5.2 and Corollary 5.3 in [7])

Proposition 5.1. Assume (H1). There exists a constant $\Lambda_{1}>0$ such that if $\lambda \leq \Lambda_{1}$, then

$$
P_{f} \leq u_{f}^{1-\alpha} .
$$

So, if $f$ is an optimal control, we have that

$$
f=\frac{\lambda}{2} u_{f}\left(1-u_{f}^{\alpha-1} P_{f}\right) .
$$

As consequence, any optimal control $f$ may be expressed as in (5.5), where the pair $\left(u_{f}, P_{f}\right):=(u, P)$ satisfies

$$
\left\{\begin{array}{l}
-\Delta u=u^{\alpha}\left(a-\frac{\lambda}{2} u+\frac{\lambda}{2} u^{\alpha} P-b u^{\beta-\alpha}\right) \text { in } \Omega \\
-\Delta P+\left(-\alpha a u^{\alpha-1}+\beta b u^{\beta-1}\right) P=\frac{\lambda}{2}\left(u-u^{\alpha} P(1+\alpha)+\alpha u^{2 \alpha-1} P^{2}\right) \text { in } \Omega \\
u=P=0 \text { on } \partial \Omega
\end{array}\right.
$$

and $u>0$. 
The former result says that, when $\lambda$ is small enough, if $f$ is an optimal control, then $\left(u_{f}, P_{f}\right)$ is a solution of (5.6). We are going to prove now that, for a range of $\lambda$, there exists a unique positive solution of (5.6) verifying $u^{1-\alpha} \geq P$ and so the unique optimal control will be

$$
f=\frac{\lambda}{2}\left(u-u^{\alpha} P\right)
$$

Theorem 5.2 (Uniqueness of optimal control). Assume $(H 1)$. There exists $\Lambda_{2}>0$ such that for $\lambda \leq \Lambda_{2}$, (5.6) possesses a unique positive solution $(u, P)$ satisfying $u^{1-\alpha} \geq P$.

Proof. We define the following map:

$$
\mathcal{T}: I:=[0, \lambda \mathcal{K}] \subset L_{+}^{\infty}(\Omega) \mapsto L_{+}^{\infty}(\Omega), \quad f \mapsto \mathcal{T}(f)=\frac{\lambda}{2}\left(u_{f}-u_{f}^{\alpha} P_{f}\right) .
$$

By Theorem 4.6, for $\lambda<\Lambda, \mathcal{T}$ is a Lipschitz continuous function with Lipschitz constant of type $\lambda \mathcal{L} / 2$, where $\mathcal{L}$ is the corresponding one for the function $f \mapsto u_{f}-$ $u_{f}^{\alpha} P_{f}$. So, we can choose $\Lambda_{2}:=\min \left\{\Lambda, \frac{2}{\mathcal{L}}\right\}$ such that for $\lambda \leq \Lambda_{2}, \mathcal{T}$ is a contractive function.

Assume that there exist two positive solutions $\left(u_{i}, P_{i}\right), i=1,2$ of (5.6) with $u_{i}^{1-\alpha} \geq P_{i}$. We define

$$
f_{i}=\frac{\lambda}{2}\left(u_{i}-u_{i}^{\alpha} P_{i}\right) \in I, \quad i=1,2
$$

Hence, by (5.6) and Proposition 3.3 we have that

$$
u_{i}=u_{f_{i}}, \quad P_{i}=P_{f_{i}}, \quad \Rightarrow \mathcal{T}\left(f_{i}\right)=f_{i} \quad i=1,2 .
$$

Since $\mathcal{T}$ is contractive, it follows that $f_{1}=f_{2}$, and again by Proposition 3.3 we have that $u_{f_{1}}=u_{f_{2}}$, hence $u_{1}=u_{2}$, and so $P_{1}=P_{2}$. This completes the proof.

Now, we use the optimal control characterization obtained by formula (5.5) to give an iterative procedure to approach it. The idea is to be near to the solution of the optimality system by sub and super solutions (see other papers related with similar problems [6], [14], [15], [17]). The interest here, besides the degeneration of second equation of the optimality system, is that we prove the convergence of the method by a different argument that used in the mentioned references. We start this part with some of notation. We define, for simplicity, the following functions,

$$
B(x, u, p)=\left[a(x)-\frac{\lambda}{2}\left(u-u^{\alpha} p\right)\right] u^{\alpha}-b(x) u^{\beta}, \text { for } x \in \Omega
$$

and, taking into account the monotony properties of the second equation of optimality system (5.6), we define

$$
D(x, u, p)= \begin{cases}\frac{\lambda}{2} u-\beta p b(x) u^{\beta-1} & \text { if } \beta<1,2 \alpha-1<0, \\ \frac{\lambda}{2} u-\beta p b(x) u^{\beta-1}+\frac{\lambda}{2} \alpha u^{2 \alpha-1} p^{2} & \text { if } \beta<1,2 \alpha-1 \geq 0, \\ \frac{\lambda}{2} u & \text { if } \beta \geq 1,2 \alpha-1<0, \\ \frac{\lambda}{2} u+\frac{\lambda}{2} \alpha u^{2 \alpha-1} p^{2} & \text { if } \beta \geq 1,2 \alpha-1 \geq 0,\end{cases}
$$


and

$$
C(x, u, p)= \begin{cases}p\left(\alpha a(x) u^{\alpha-1}-\frac{\lambda}{2} u^{\alpha}(\alpha+1)\right)+\frac{\lambda}{2} \alpha u^{2 \alpha-1} p^{2} & \text { if } \beta<1,2 \alpha-1<0, \\ p\left(\alpha a(x) u^{\alpha-1}-\frac{\lambda}{2} u^{\alpha}(\alpha+1)\right) & \text { if } \beta<1,2 \alpha-1 \geq 0, \\ p\left(\alpha a(x) u^{\alpha-1}-\beta b(x) u^{\beta-1}-\right. & \\ \left.-\frac{\lambda}{2} u^{\alpha}(\alpha+1)\right)+\frac{\lambda}{2} \alpha u^{2 \alpha-1} p^{2} & \text { if } \beta \geq 1,2 \alpha-1<0, \\ p\left(\alpha a(x) u^{\alpha-1}-\beta b(x) u^{\beta-1}-\frac{\lambda}{2} u^{\alpha}(\alpha+1)\right) & \text { if } \beta \geq 1,2 \alpha-1 \geq 0 .\end{cases}
$$

We are interested in the solutions, $(u, p)$, of optimality system (5.6) that satisfy $u_{\lambda \mathcal{K}} \leq u \leq u_{0}$ and $0 \leq p \leq \lambda \psi$ (recall (5.3)). Consequently, we can reduce the study for solutions that satisfy $(u, p) \in\left[u_{\lambda \mathcal{K}}, u_{0}\right] \times[0, \lambda \psi]$. Therefore, there exist a constant $K>0$ and a function $M_{1}(x), x \in \Omega$, satisfying hypothesis $(H M)$, such that

$$
\begin{array}{ll}
B(x, u, p)+K u^{\alpha} & (\nearrow u, \nearrow p), \\
C(x, u, p)+M_{1}(x) p & (\searrow u, \nearrow p), \\
D(x, u, p)+M_{1}(x) p & (\nearrow u, \nearrow p),
\end{array}
$$

i. e., $B(x, u, p)+K u^{\alpha}$ is increasing in $u$ for fixed $x \in \Omega$ and $0 \leq p \leq \lambda \psi$ and increasing in $p$ for fixed $x \in \Omega$ and $u_{\lambda \mathcal{K}} \leq u \leq u_{0}$. The other terms are interpreted analogously.

DEFINITION 5.3 (Sub-super solutions). The functions $u_{1}, p_{1}, u^{1}, p^{1} \in L^{\infty}(\Omega) \cap$ $H^{1}(\Omega)$ are said to be a system of sub-super solutions for the optimality system (5.6), if they verify

$$
\left\{\begin{array}{c}
u_{1}(x) \leq u^{1}(x), \quad p_{1}(x) \leq p^{1}(x), \quad \text { a.e. in } \Omega, \\
p_{1} \leq 0 \leq p^{1}, \quad \text { on } \partial \Omega
\end{array}\right.
$$

and there exists a positive constant $k$ such that

$$
0<k d_{\Omega}(x) \leq u_{1}(x) \leq u^{1}(x), \quad \text { a.e. in } \Omega
$$

and, for any $\phi \in H_{0}^{1}(\Omega), \phi \geq 0$,

$$
\begin{gathered}
\int_{\Omega} \nabla u^{1} \cdot \nabla \phi \geq \int_{\Omega} B\left(x, u^{1}, p^{1}\right) \phi, \quad \int_{\Omega} \nabla u_{1} \cdot \nabla \phi \leq \int_{\Omega} B\left(x, u_{1}, p_{1}\right) \phi \\
\int_{\Omega} \nabla p^{1} \cdot \nabla \phi \geq \int_{\Omega} C\left(x, u_{1}, p^{1}\right) \phi+\int_{\Omega} D\left(x, u^{1}, p^{1}\right) \phi \\
\int_{\Omega} \nabla p_{1} \cdot \nabla \phi \leq \int_{\Omega} C\left(x, u^{1}, p_{1}\right) \phi+\int_{\Omega} D\left(x, u_{1}, p_{1}\right) \phi .
\end{gathered}
$$

Recall that a function $v \in H^{1}(\Omega)$ is said to be less or equal than $w \in H^{1}(\Omega)$ on $\partial \Omega$ when $(v-w)^{+}=\max \{0, v-w\} \in H_{0}^{1}(\Omega)$.

It is not difficult to prove that, under the hypothesis of Theorem 5.2, there exists a $\Lambda_{3}>0$, such that, if $\lambda \leq \Lambda_{3}$, then the functions

$$
u_{1}=u_{\lambda \mathcal{K}}, \quad p_{1} \equiv 0, \quad u^{1}=u_{0}, \quad p^{1}=\lambda \psi,
$$

are a system of sub-super solutions for the optimality system (5.6) in the sense of Definition 5.3. We show only the case $p^{1}=\lambda \psi$ when $\beta \geq 1,2 \alpha-1 \geq 0$. The other cases are similar. It is not hard to show that $p^{1}=\lambda \psi$ is a supersolution if

$$
\lambda\left(\mathcal{K}-m_{\lambda_{1}} \psi\right) \geq \lambda \psi\left[\alpha a u_{\lambda \mathcal{K}}^{\alpha-1}-\beta b u_{\lambda \mathcal{K}}^{\beta-1}-\frac{\lambda}{2} u_{\lambda \mathcal{K}}^{\alpha}(\alpha+1)\right]+\frac{\lambda}{2} u_{0}+\frac{\lambda}{2} \alpha u_{0}^{2 \alpha-1}(\lambda \psi)^{2}
$$


or equivalently,

$$
\mathcal{K} \geq \psi\left[m_{\lambda_{1}}+\alpha a u_{\lambda \mathcal{K}}^{\alpha-1}-\beta b u_{\lambda \mathcal{K}}^{\beta-1}\right]-\frac{\lambda}{2} u_{\lambda \mathcal{K}}^{\alpha}(\alpha+1) \psi+\frac{1}{2} u_{0}+\frac{\alpha}{2} \lambda^{2} u_{0}^{2 \alpha-1} \psi^{2} .
$$

Recalling the definition of $m_{\lambda_{1}}$, for $\lambda \leq \lambda_{1}$ we have that $m_{\lambda_{1}}+\alpha a u_{\lambda \mathcal{K}}^{\alpha-1}-\beta b u_{\lambda \mathcal{K}}^{\beta-1} \leq 0$, and by (3.5) $u_{0} \leq \mathcal{K}$, it is enough to take $\lambda$ small to obtain that $p^{1}$ is a supersolution.

Now, we define by induction, for $n \geq 2$, the sequences $\left\{u_{n}\right\},\left\{u^{n}\right\},\left\{p_{n}\right\},\left\{p^{n}\right\} \in$ $H_{0}^{1}(\Omega)$, as

Observe that sequences $\left\{u_{n}\right\},\left\{u^{n}\right\}$, are well defined because the map $u \mapsto K u^{\alpha}$ is continuous and strictly increasing and such that $B(x, u, p)+K u^{\alpha}$ is also increasing in $u$, when the other variables are fixed. (See more details in [5], [10]).

On the other hand, fixed $u_{1}, u^{1}$ and thanks to (5.8), the problems (5.12) and (5.13) are in the setting of (2.4) and so by Theorem 2.5 it follows the existence and uniqueness of $p_{2}$ and $p^{2}$ and such that $p_{2} \leq p^{2}$ and so on. We note that for (5.10)-(5.11) and (5.12)-(5.13), the sub-super solutions method works (cf. [12]). The standard method gives us the following order relation

$$
\begin{aligned}
& u_{1} \leq u_{2} \leq \ldots \leq u_{n} \leq u^{n} \leq u^{n-1} \leq \ldots \leq u^{1}, \\
& p_{1} \leq p_{2} \leq \ldots \leq p_{n} \leq p^{n} \leq p^{n-1} \leq \ldots \leq p^{1},
\end{aligned}
$$

and

$$
u_{n} \nearrow u, u^{n} \searrow v, p_{n} \nearrow p, p^{n} \searrow q,
$$

(pointwise), where $u, v, p, q$ belong to $C^{1, \delta}(\Omega)$, for any $\delta \in(0,1)$, and satisfy the system

$$
\begin{cases}-\Delta u=B(x, u, p) & \text { in } \Omega, \\ -\Delta v=B(x, v, q) & \text { in } \Omega, \\ -\Delta p=C(x, v, p)+D(x, u, p) & \text { in } \Omega, \\ -\Delta q=C(x, u, q)+D(x, v, q) & \text { in } \Omega, \\ u=v=p=q=0, & \text { on } \partial \Omega\end{cases}
$$

and

$$
\begin{gathered}
u_{1}=u_{\lambda \mathcal{K}} \leq u, v \leq u^{1}=u_{0} \quad \text { in } \Omega \\
p_{1}=0 \leq p, q \leq p^{1}=\lambda \psi \leq u_{\lambda \mathcal{K}}^{1-\alpha} \quad \text { in } \Omega .
\end{gathered}
$$

Clearly, if $(u, p)$ is the solution of the optimality system (5.6), then $(u, u, p, p)$ is a solution of (5.14). So, to complete the iterative approximation and the convergence of the sequences $\left\{u_{n}\right\},\left\{u^{n}\right\},\left\{p_{n}\right\},\left\{p^{n}\right\}$ to the unique solution, $(u, p)$, of the optimality system, it is sufficient to prove the uniqueness of the solution for the system (5.14), under the conditions (5.15). To do it, we need the following technical lemma.

Lemma 5.4. Assume (H1). Then

$$
\forall f, g \in[0, \lambda \mathcal{K}] \subset L_{+}^{\infty}(\Omega),
$$


it is possible to define the function $P_{f, g}$ as the unique positive solution of the problem

$$
\begin{cases}-\Delta P=C\left(x, u_{f}, P\right)+D\left(x, u_{g}, P\right), & \text { in } \Omega \\ P=0, & \text { on } \partial \Omega,\end{cases}
$$

satisfying

$$
0 \leq P_{f, g} \leq \lambda \psi
$$

provided that the parameter $\lambda$ is small enough and the function $\psi$ is defined in (5.2). Moreover, the map defined before, $(f, g) \in[0, \lambda \mathcal{K}] \times[0, \lambda \mathcal{K}] \mapsto P_{f, g} \in L^{\infty}(\Omega)$ is Lipschitz continuous.

Analogous result is obtained interchanging $u_{f}$ and $u_{g}$ in (5.16).

Proof. We consider the case $\beta \geq 1,2 \alpha-1 \geq 0$. The other cases have similar proofs. Observe that, in this case, equation (5.16) is

$$
\left\{\begin{array}{l}
-\Delta P+\left[-\alpha a u_{f}^{\alpha-1}+\beta b u_{f}^{\beta-1}+\frac{\lambda}{2} u_{f}^{\alpha}(1+\alpha)\right] P=\frac{\lambda}{2} u_{g}+\frac{\lambda}{2} \alpha u_{g}^{2 \alpha-1} P^{2} \text { in } \Omega \\
P=0 \quad \text { on } \partial \Omega
\end{array}\right.
$$

Taking into account the Theorem 2.5, the condition (5.17) and the definition of the function $\psi$, we can use the sub-super solution method with $p_{*} \equiv 0$ as subsolution and $p^{*} \equiv \lambda \psi$ as supersolution, provided $\lambda>0$ small. Thus, the existence of positive solution of (5.18) is proved. The uniqueness follows of a contradiction argument. Suppose that $P, Q$ are two solutions under above requirements, then $P-Q$ satisfies

$$
\left(-\Delta+M_{1}(x)\right)(P-Q)=0,
$$

where

$$
M_{1}=-\alpha a u_{f}^{\alpha-1}+\beta b u_{f}^{\beta-1}+\frac{\lambda}{2} u_{f}^{\alpha}(1+\alpha)-\frac{\lambda}{2} \alpha u_{g}^{2 \alpha-1}(P+Q) .
$$

Observe that $M_{1}$ satisfies (HM). Now, using that $P, Q \leq \lambda \psi$, we obtain that

$$
M_{1} \geq-\alpha a u_{T_{\lambda}}^{\alpha-1}+\beta b u_{T_{\lambda}}^{\beta-1}+\frac{\lambda}{2} u_{T_{\lambda}}^{\alpha}(1+\alpha)-\lambda^{2} \alpha u_{0}^{2 \alpha-1} \psi
$$

and so we can prove the existence of a function $N$ satisfying (HM) and $\lambda_{0}>0$ such that for $\lambda \leq \lambda_{0}$

$$
M_{1} \geq N \quad \text { in } \Omega \text { and } \quad \sigma_{1}(-\Delta+N)>0 .
$$

Hence, the previous equation has the unique solution $(P-Q) \equiv 0$.

To show the lipschitzian character of the map $(f, g) \mapsto P_{f, g}$, let $P_{f, g}$ be the solution of problem (5.18) satisfying (5.17). Denote $\bar{q}=P_{\bar{f}, \bar{g}}$ and $q=P_{f, g}$. Then, some calculus give

$$
\begin{aligned}
& (-\Delta+M(x))(q-\bar{q})=R_{f, \bar{f}, g, \bar{g}}:=\alpha a q\left(u_{f}^{\alpha-1}-u_{\bar{f}}^{\alpha-1}\right)- \\
& \beta b q\left(u_{f}^{\beta-1}-u_{\bar{f}}^{\beta-1}\right)-\frac{\lambda}{2}(\alpha+1) q\left(u_{f}^{\alpha}-u_{\bar{f}}^{\alpha}\right)+\frac{\lambda}{2}\left(u_{g}-u_{\bar{g}}\right)+\frac{\lambda}{2} \alpha q^{2}\left(u_{g}^{2 \alpha-1}-u_{\bar{g}}^{2 \alpha-1}\right),
\end{aligned}
$$

where

$$
M=-\alpha a u_{\bar{f}}^{\alpha-1}+\beta b u_{\bar{f}}^{\beta-1}+\frac{\lambda}{2}(\alpha+1) u_{\bar{f}}^{\alpha}-\frac{\lambda}{2} \alpha(q+\bar{q}) u_{\bar{g}}^{2 \alpha-1}
$$


As in the proof of (5.19), we can prove the existence of a function $N$ satisfying (HM) such that for $M \geq N$ in $\Omega$ and $\sigma_{1}(-\Delta+N)>0$ for small $\lambda$. Thus, by Theorem 2.5 we get that

$$
\|q-\bar{q}\|_{\infty}=\left\|P_{f, g}-P_{\bar{f}, \bar{g}}\right\|_{\infty} \leq\left\|P_{f, g}-P_{\bar{f}, \bar{g}}\right\|_{C^{1}(\bar{\Omega})} \leq C\left\|R_{f, \bar{f}, g, \bar{g}}\right\|_{\infty}
$$

Now, we can use a similar argument to the used in the proof of Theorem 4.6 to obtain a bound of $\|T(f, g)\|_{\infty}$, we have

$$
\begin{aligned}
& \left\|\alpha a q\left(u_{f}^{\alpha-1}-u_{\frac{\alpha}{f}}^{\alpha-1}\right)+\beta b q\left(u_{f}^{\beta-1}-u_{\bar{f}}^{\beta-1}\right)+\frac{\lambda}{2}(\alpha+1) q\left(u_{f}^{\alpha}-u_{\frac{\alpha}{f}}^{\alpha}\right)\right\|_{\infty} \leq C\|f-\bar{f}\|_{\infty}, \\
& \left\|\frac{\lambda}{2}\left(u_{g}-u_{\bar{g}}\right)+\frac{\lambda}{2} \alpha q^{2}\left(u_{g}^{2 \alpha-1}-u_{\bar{g}}^{2 \alpha-1}\right)\right\|_{\infty} \leq C\|g-\bar{g}\|_{\infty},
\end{aligned}
$$

and so, $\left\|R_{f, \bar{f}, g, \bar{g}}\right\|_{\infty} \leq C\left\{\|f-\bar{f}\|_{\infty}+\|g-\bar{g}\|_{\infty}\right\}$. Finally, we have

$$
\left\|P_{f, g}-P_{\bar{f}, \bar{g}}\right\|_{\infty} \leq C\left\{\|f-\bar{f}\|_{\infty}+\|g-\bar{g}\|_{\infty}\right\},
$$

for a convenient positive constant $C$.

THEOREM 5.5. Assume (H1). There exists a positive constant $\Lambda_{4}$, such that, if $\lambda \leq \Lambda_{4}$, then the system (5.14)-(5.15) has a unique solution.

Proof. The main idea is simple. We will use the lipschitzian character of the solutions of the system (5.14)-(5.15) with respect to the controls and an argument similar to the used in Theorem 5.2.

Suppose $\left(u_{i}, v_{i}, p_{i}, q_{i}\right)$, for $i=1,2$, are two solutions of the system (5.14)-(5.15). We define, for $i=1,2$,

$$
f_{i}=\frac{\lambda}{2}\left[u_{i}-u_{i}^{\alpha} p_{i}\right] \quad g_{i}=\frac{\lambda}{2}\left[v_{i}-v_{i}^{\alpha} q_{i}\right]
$$

Now, taking into account the previous notation, we have for $i=1,2$,

$$
u_{i}=u_{f_{i}}, \quad v_{i}=u_{g_{i}}, \quad p_{i}=P_{g_{i}, f_{i}}, \quad q_{i}=P_{f_{i}, g_{i}}
$$

and

$$
f_{i}=\frac{\lambda}{2}\left[u_{f_{i}}-u_{f_{i}}^{\alpha} P_{g_{i}, f_{i}}\right] \quad g_{i}=\frac{\lambda}{2}\left[u_{g_{i}}-u_{g_{i}}^{\alpha} P_{f_{i}, g_{i}}\right] .
$$

We know, recall Theorem 4.6 and Lemma 5.4 , that the operator $T:[0, \lambda \mathcal{K}] \times[0, \lambda \mathcal{K}] \rightarrow$ $L^{\infty}(\Omega) \times L^{\infty}(\Omega)$, defined as,

$$
T(f, g)=\left(\frac{\lambda}{2}\left[u_{f}-u_{f}^{\alpha} P_{g, f}\right], \frac{\lambda}{2}\left[u_{g}-u_{g}^{\alpha} P_{f, g}\right]\right),
$$

is Lipschitz continuous, with constant $\lambda C / 2$, where $C>0$ is the Lipschitz constant of $\operatorname{map}(f, g) \mapsto\left(u_{f}-u_{f}^{\alpha} P_{f, g}, u_{g}-u_{g}^{\alpha} P_{g, f}\right)$ and verifies $T\left(f_{i}, g_{i}\right)=\left(f_{i}, g_{i}\right)$. Therefore, by taking $\lambda<\min \left\{\Lambda_{1}, \frac{2}{C}\right\}, T$ is a contraction and consequently has an unique fixed point. So, $\left(f_{1}, g_{1}\right)=\left(f_{2}, g_{2}\right)$. Then, we have $u_{1}=u_{2}, v_{1}=v_{2}$ and finally $p_{1}=p_{2}$ and $q_{1}=q_{2}$. 
Acknowledgments. M. Delgado and A. Suárez thank to the Spanish Ministry of Science and Technology under grant BFM2000-0797. and J. A. Montero to "Junta de Andalucía" (FQM116) and DGESIC (PB98-1343) by the partial financial support to the elaboration of this work.

\section{REFERENCES}

[1] D.G. Aronson And L.A. Peletier, Large time behaviour of solutions of the porous medium equation in bounded domains, J. Diff. Eqs., 39 (1981), pp. 378-412.

[2] C. Bandle, M. A. Pozio And A. Tesei, The asymptotic behavior of the solutions of degenerate parabolic equations, Trans. A.M.S., 303 (1987), pp. 487-501.

[3] H. Berestycki, L. Nirenberg and S.R.S. VARAdHAn, The principal eigenvalue and maximum principle for second order elliptic operators in general domains, Comm. Pure Appl. Math., XLVII (1994), pp. 47-92.

[4] M. Bertsch and R. Rostamian, The principle of linearized stability for a class of degenerate diffusion equations, J. Diff. Eqns., 57 (1985), pp. 373-405.

[5] A. CAÑADA AND J.L. GÁmEZ, Existence of solutions for some semilinear degenerate elliptic systems with applications to populations dynamics, Diff. Eqns. and Dyn. Sist., 3 (1995), pp. 189-204.

[6] A. CAÑAda, J.L. Gámez And J.A. Montero, Study of an optimal control problem for diffusive nonlinear elliptic equations of logistic type, SIAM J. Control Optim., 36 (1998), pp. 11711189.

[7] M. Delgado, J.A. Montero and A. SuÁrez, Optimal control for the degenerate elliptic logistic equation, Appl. Math. Optim., 45 (2002), pp. 325-345.

[8] M. Delgado And A. SuÁrez, On the existence of dead cores for degenerate Lotka-Volterra models, Proc. Royal Society of Edin. A, 130 (2000), pp. 743-766.

[9] - On the structure of the positive solutions of the logistic equation with nonlinear diffusion, J. Math. Anal. Appl., 268 (2002), pp. 200-216.

[10] J.I. DíAz , Nonlinear partial differential equations and free boundaries, Vol I Elliptic equations, London, Pitman 1985.

[11] M.E. Gurtin And R.C. MaCCamy, On the diffusion of biological populations, Math. Biosci., 33 (1977), pp. 35-49.

[12] J. Hernández, F. MAncebo And J.M. Vega de PradA, On the linearization of some singular nonlinear elliptic problems and applications, Ann. Inst. H. Poincare Anal. Non-Lineaire, 19 (2002), pp. 777-813.

[13] A. Kufner, Weighted Sobolev Spaces Text zur Mathematik, 31, Teubner, Leipzig (1980).

[14] S. Lenhart, V. Protopopescu and S. Stojanovic, A two-sided game for nonlocal competitive systems with control on source terms, IMA Vol. Math. Appl., 53 (1993), Springer, New York, pp. 135-152.

[15] A.W. Leung, Optimal harvesting-coefficient control of steady-state prey-predator diffusive Volterra-Lotka systems, Appl. Math. Optim., 31 (1995), pp. 219-241.

[16] - Positive solutions for systems of PDE and optimal control, Nonlinear Analysis, 47 (2001), pp. 1345-1356.

[17] A.W. Leung And S. Stojanovic, Optimal control for elliptic Volterra-Lotka type equations, J. Math. Anal. Appl., 173 (1993), pp. 603-619.

[18] J.A. Montero, A uniqueness result for an optimal control problem on a diffusive elliptic Volterra-Lotka type Equation, J. Math. Anal. Appl., 243 (2000), pp. 13-31.

[19] M.A. Pozio AND A. TESEI, Support properties of solutions for a class of degenerate parabolic problems, Comm. Part. Diff. Eqns., 12 (1987), pp. 47-75.

[20] S. StojAnovic, Modeling and minimization of extinction in Volterra-Lotka type equations with free boundaries, J. Diff. Eqns., 134 (1997), pp. 320-342. 